\title{
Implementation of new technique for Face recognition based on quantum computing
}

\author{
Amjed yousif sahib,Email:- Amjad_yousf@yahoo.com
}

\begin{abstract}
In this research, a new technique for measuring facial recognition face similarity based on quantum computing (oracle) has been proposed. This technique is transforming the oracle of Grover's search algorithm into correlation oracle with feeding image. The implementation is carried out on a classical computer with Matlab. The improvement in this work came from combination between quantum Grover's algorithm and classical face recognition algorithm where, the number of steps require in Grover's to find target image is $\frac{\pi}{4} \sqrt{N}$ which is exponential improvement as compared with $\frac{N}{2}$ steps for classical case. This reduction of steps reduces the time required to process and the complexity and enables us to increase the amount of information to be processed. The quantum phenomena such as superposition, entanglement and vector space are formulated to represent quantum information and quantum processes on the classical computer. Public available AT and T Laboratories in university of Cambridge database are tested on the proposed algorithm.
\end{abstract}

Keyword: Quantum computing, image processing, Matlab

في هذا البحث ,تم اقتراح طريقة جديدة لقياس التشابه في الوجوه بالاعتماد على الحاسوب الكمي(الدالة

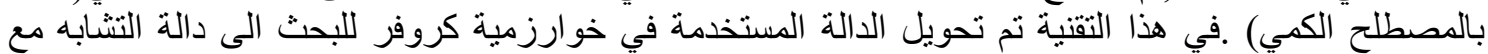

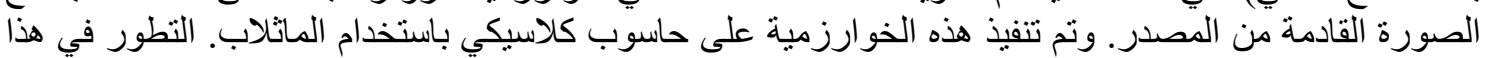

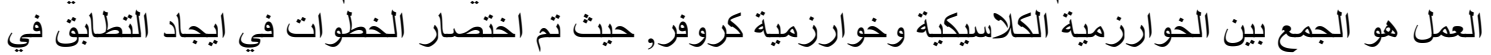

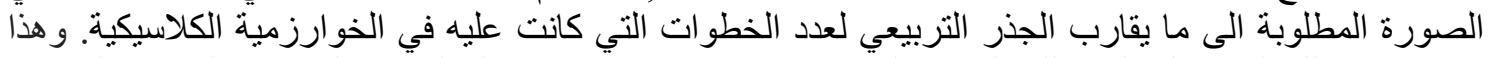

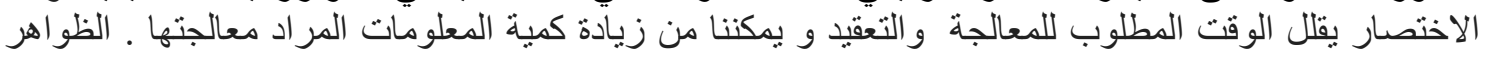

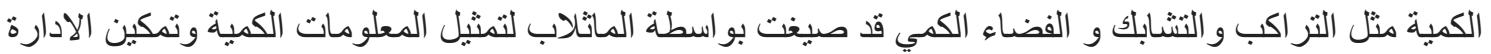

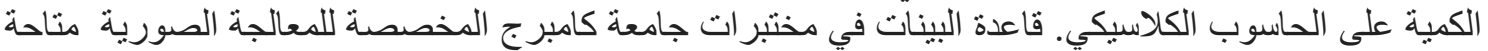
للعامة قد اختبرت على الخو ارزمية المقترحة. 


\section{Introduction}

Facial recognition is important topic now a day, especially for security purposes. Some of crimes happened in repetitive act. Use surveillance data can be vital for imitate criminals activities. Facial recognition one of what so called biometric technology [1]. Many algorithm where proposed in this field advantaging classical computers evolution. In our universe there are huge amount of information need to be managed, or what called big data systems. In order to achieve this goal (management), quantum computing technique is a valuable option that could manage the large amount of information fast, accurate, and efficient; theoretically at least. Quantum computation may be defined as performing any process on a given information using laws of quantum mechanics [2]. In this case data are considered in terms of quantum states which are manipulated by certain quantum gates using a predetermined quantum algorithm. Searching is an important task, and there are varieties classical algorithms proposed, and practically applied successfully. In classical regime the search is done systematically check all the possibilities to find the desired solution. Clearly if search spaece contain $\mathrm{N}$ entries, then the iteration to complete a search is on average $\mathrm{N} / 2$ [3]. In the scope of previous concepts, classical way of thinking bounded for no more than that. Quantum computation methods used parallelism, making it superior; which will we discussed later in the current work. The idea of this work is to convert the classical image (in the work 0 and 32 images) using a classical interface with quantum that build with Mat lab, where the internal representation of images is given. All this processes to that quantum data inside done in classical computer. The results are measured by using quantum interface in the algorithm and detect the classical outcome data.

\section{Quantum image processing background:}

It is new field and till now this branch is still under experimenting. However, quantum image processing discipline begun with Vlasov at 1997. In his work he use quantum computation to recognize the orthogonally of image [4]. Schutzhold in 2002, proposed quantum binary images algorithm for locating specific patterns [5]. Beach, Lomont, and Cohen in October 2003, gave demonstration of using quantum algorithms (the most uses is Grover's algorithm) in image processing. In their study, they explained a method that a quantum algorithm is used to detect a placement, of a specific target. In that paper they state the importance of quantum image process and how it will be useful role in the wartime [6]. The study continue with, venegas-andraca[7] proposed quantum image representation such as Qubit lattice[8,9]. A first Ph.D. thesis in this branch. A new approach given by the proposed work of Le et al[10], they provide a flexible exemplification a for images as normalized states on a quantum computer which apprehend the colors and positions information in the target images, this was important point in quantum image branch. Till now different researcher's and their 
different ideas try to presenting a new techniques of the quantum image processing[11-15].

\section{Flow chart implementation:}

The diagrammatic representation shown in figure(1) illustrates our algorithm process. Where the quantum image process are analyzed then this steps were implemented with Matlab.

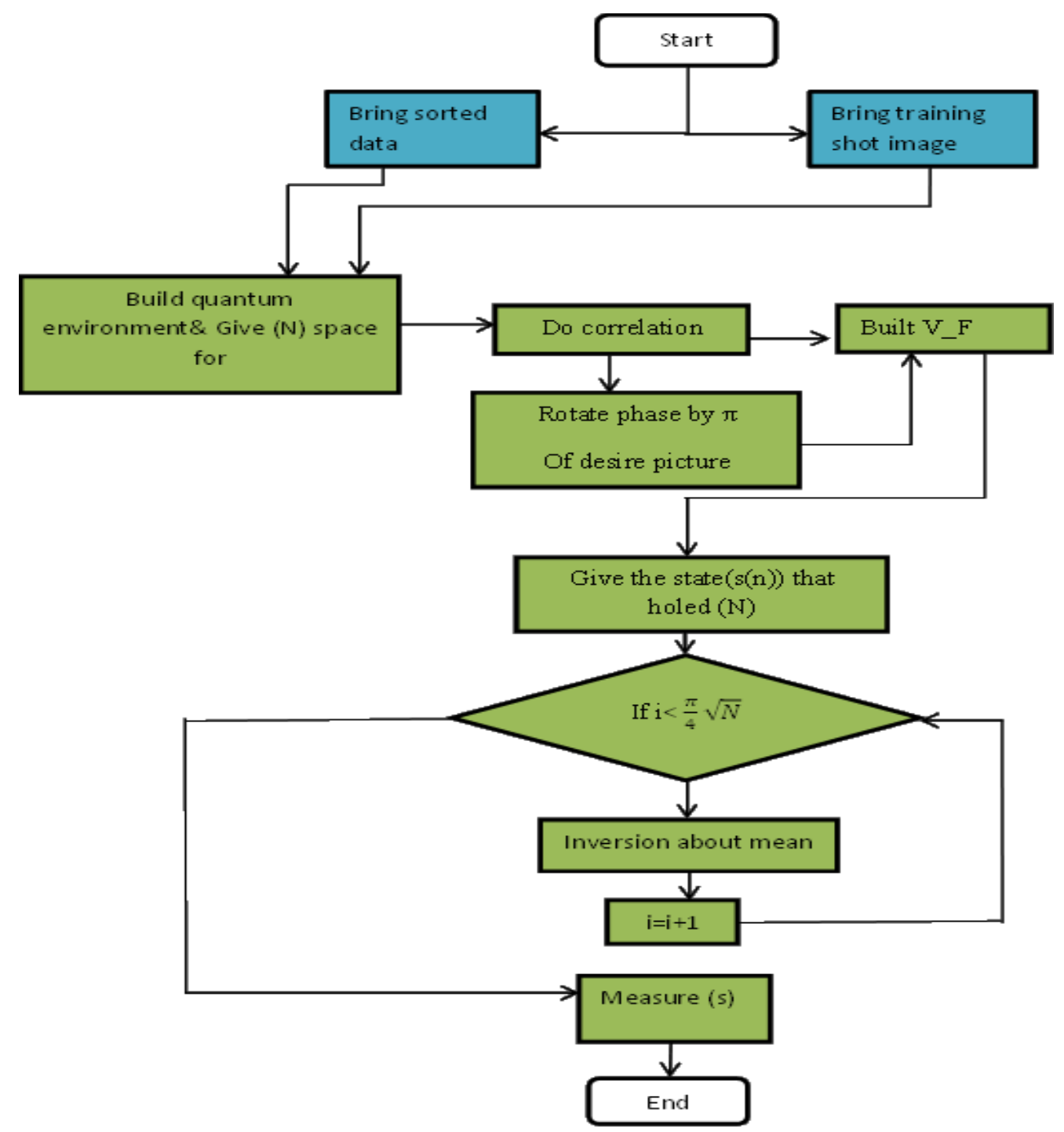

Fig. 1 flow chart of quantum image processing 


\section{Fundamentals of Quantum computation and interface with classical:}

\section{Superposition:}

The unite of measure classical information is bit. Analogous quantum bit (Qbit) is used for quantum information measurement. The physics difference between two units is the the quantum bits (Qbit) involved states other than zero or one. May also group of the states, which is often called superposition's:

$$
|\Psi\rangle=\alpha|0\rangle+\beta|1\rangle
$$

Where $(\alpha)$ and $(\beta)$ are the probability amplitude of superposed states and

$$
\alpha^{2}+\beta^{2}=1 \text {. }
$$

The states $|0\rangle$ and $|1\rangle$ named computational basis states and the vector repsantation of this orthonormal basis given as

This is built in mat lab as:

$$
|0\rangle=\left[\begin{array}{l}
1 \\
0
\end{array}\right] \text { and }|1\rangle=\left[\begin{array}{l}
0 \\
1
\end{array}\right]
$$

-Function psi (b) = bin2vec ('number of bin')

$-\operatorname{dec}=\operatorname{bin} 2$ dec ('number of bin')

-psi (v) = dec2vec (dec,length(number of bin')

For example three qubit the vector hold seven states at time

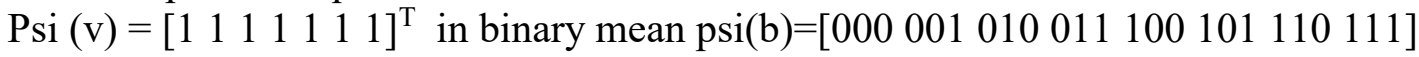

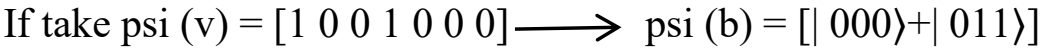

\section{Function implementation of face recognition based on quantum computation:}

This implanted based on the quantum Grover algorithm the idea is the phase of desire state changed. After build the unitary matrix of data bias for images then, we do correlation between shoot image frame form cameras with sorted images.

\subsection{Built unitary matrix:}

In this work an available data from Laboratories Cambridge [17]. The data contents (10) different images with different angle also the expression is different for about (40) persons face. This details increase algorithm capability during the test. The face data is shown in Figure (2). 


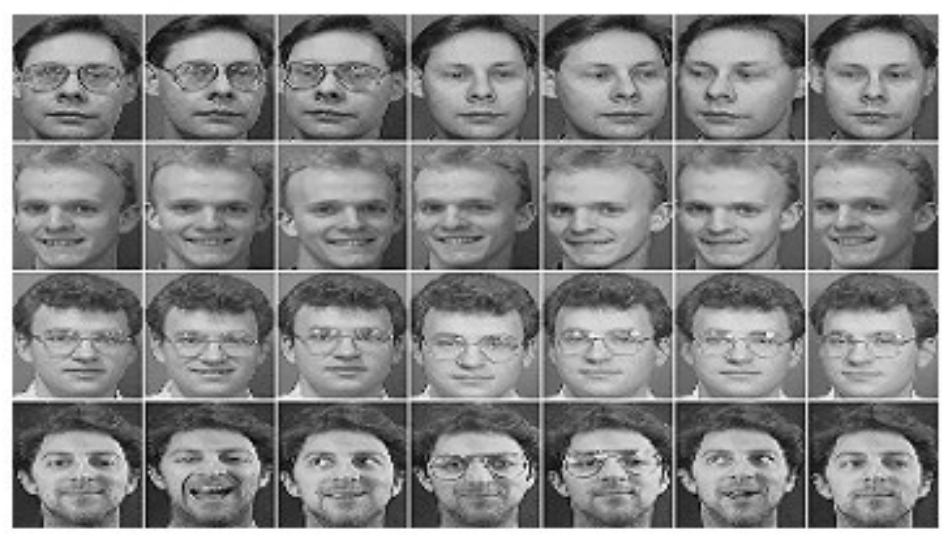

Fig.(2) AT and T database

In this work 5 qubits considered and up to 32 images are chosen $\left(2^{5}=32\right)$. The classical data convert to the quantum data as unitary matrix as in figure (3).

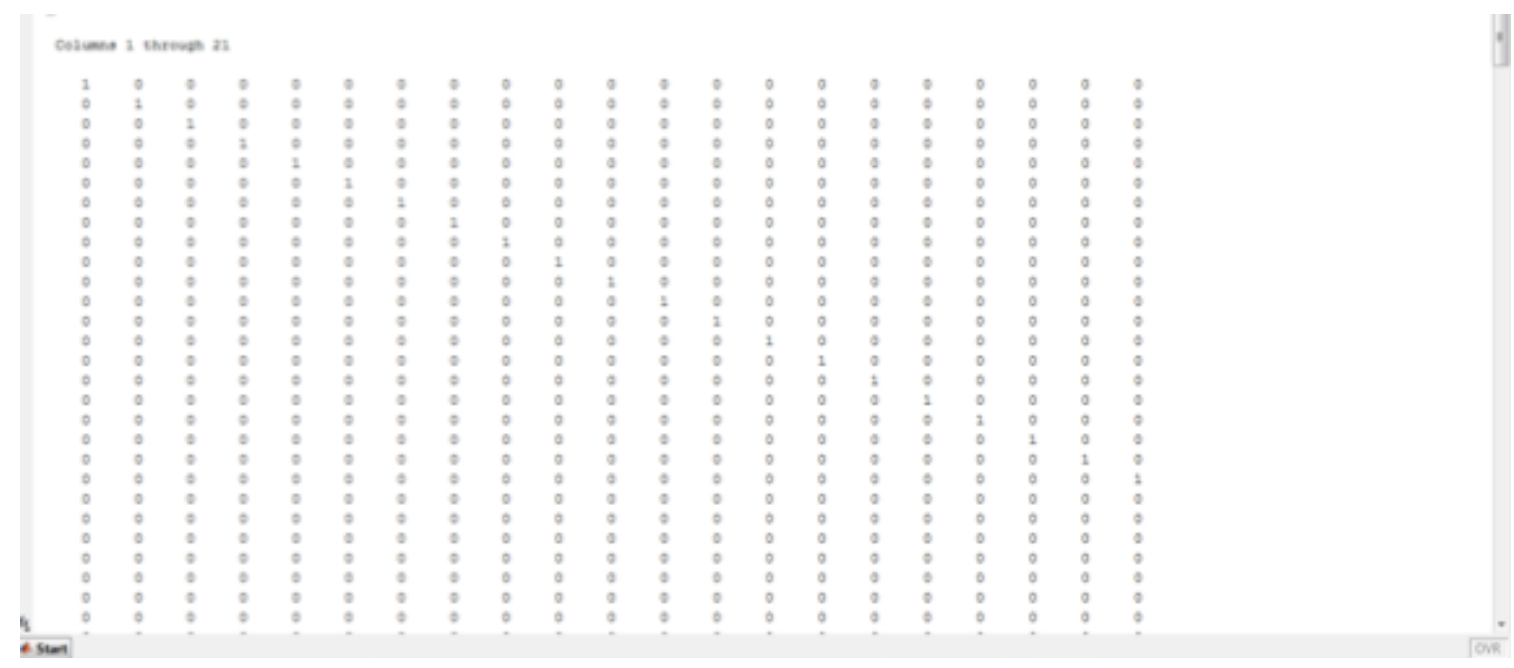

Fig.3 unitary matrix of data base

\subsection{Hadmard :}

Hadmard gate transform the quibit basis state into superposition state. This implemented in the Matlab as in figure (4) 
(a)

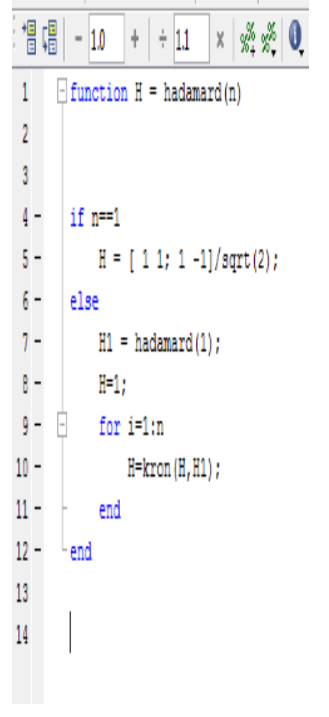

(b)

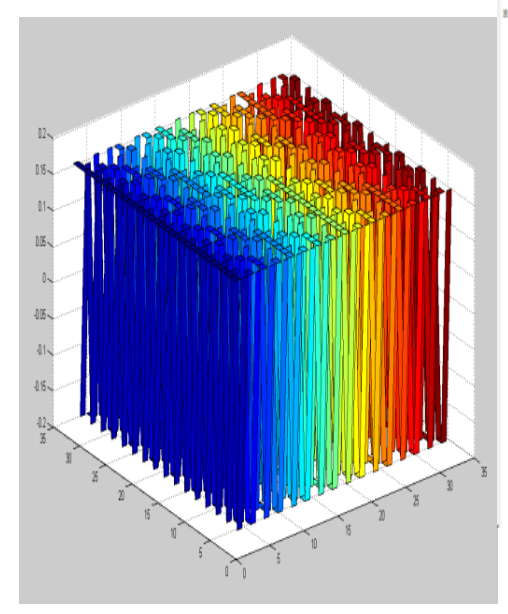

(c)

fig.4 Hadmard implementation with Matlab: (a) the program in Matlab (b) show that Hadmard cover all 32 images database (C) the matrix output of $\left(2^{5}\right)$ of Hadmard gate

\subsection{Apply oracle (correlation function):}

The applying of the oracle (function in classical rgime), mean changing of phase of the desired state, this can be expressed in mathematic by changing the sign of the marked states

$$
|\psi\rangle=\frac{1}{\sqrt{2^{n}}} \sum_{x=0}^{2^{n}-1}(-1)^{f(x)}|x\rangle .
$$

the briefly steps explain how this can be done with matlab .

\#image from data

-M= imread (imagename1)

\#tested image this command in matalb read the image

$-\mathrm{C}=$ imread (imagname2)

\#check if it the desired image by doing the correlation is done with matlab command as follow

$-\mathrm{R}=\operatorname{corr} 2(\mathrm{M}, \mathrm{C})$

\#insert the sign result from correlation in global data

$-V_{-} f(i+1, i+1)=-1^{\wedge}(f e v a l(f, R)$.

These commend change the sign of desired state in to negative which mean in quantum changing the phase. Figure (5) shows the result of the above state. 
(a)

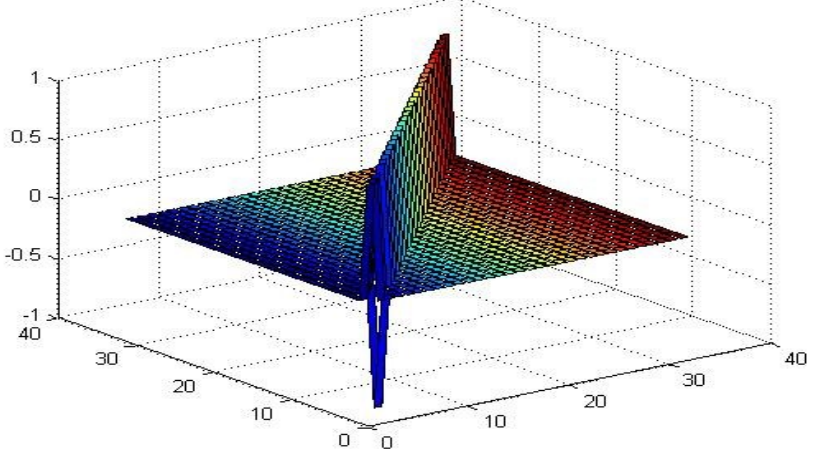

(b)

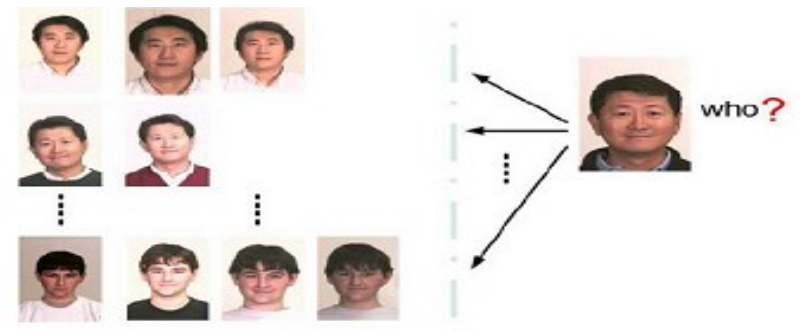

Fig.5 the correlation function : (a) the marked state is changed in sign (b) search target image in the database

\subsection{Inversion about average:}

The performing a unitary operator called inversion about the mean, will increase the probability of marked state. After that repeat apply the oracle then inversion about mean for number of iteration till reach the maximum probability of marked state. Fig(6) shorrs Matlab implementation progress.

(a)

(b)

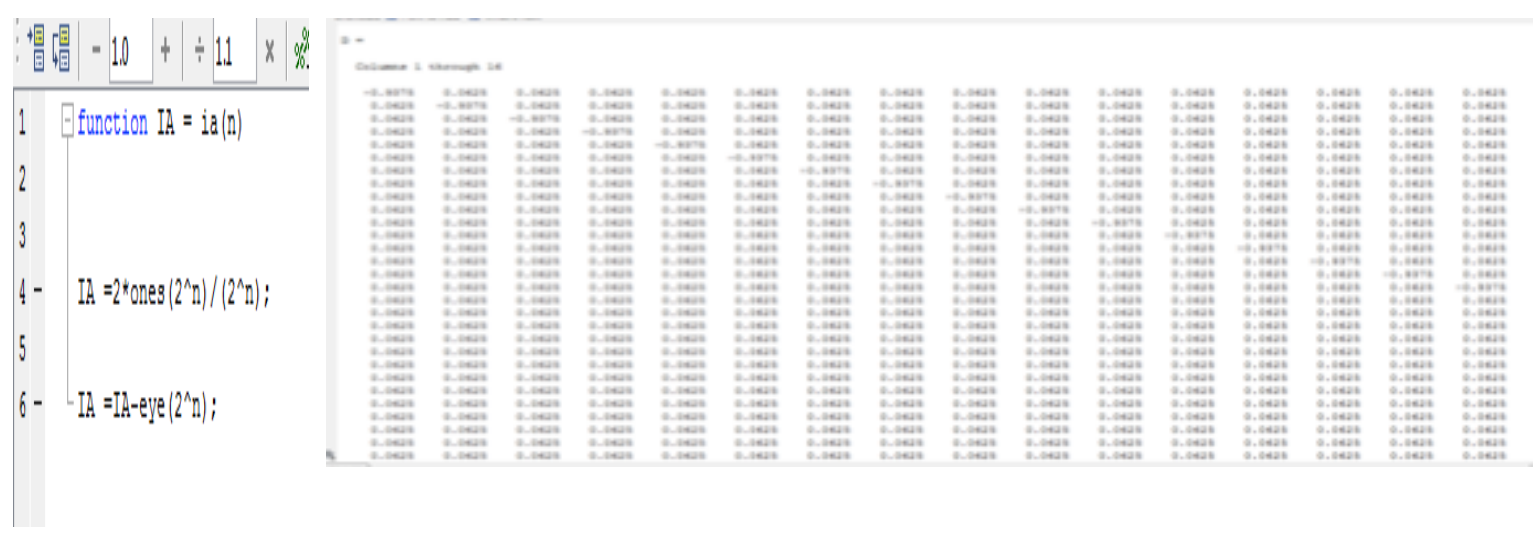

Figure(6): (a) implementation of inversion about the mean in Mata lab (b) Matrix output of inversion about mean .

\section{Discussion:}

As the quantum processing adopted, to optimize facial recognition, for big data search. Quantum processing efficiency shows its superiority when dealing with massive information. However, processing small data shows approach of classical to quantum. Parallelisms are a phenomenal advance in computing techniques, adding to other 
quantum property which is entanglement. Entanglement gives quantum computers the ability to works beyond known orthodox physical communications systems. The accessing database is remotely with liberty of geo-aspects and optimized temporal factors. Figure (7) shows the difference of speed in processing with respect to number of iteration. The difference in iteration is not large because of the number of qubits used are only five in this work. 
(a)

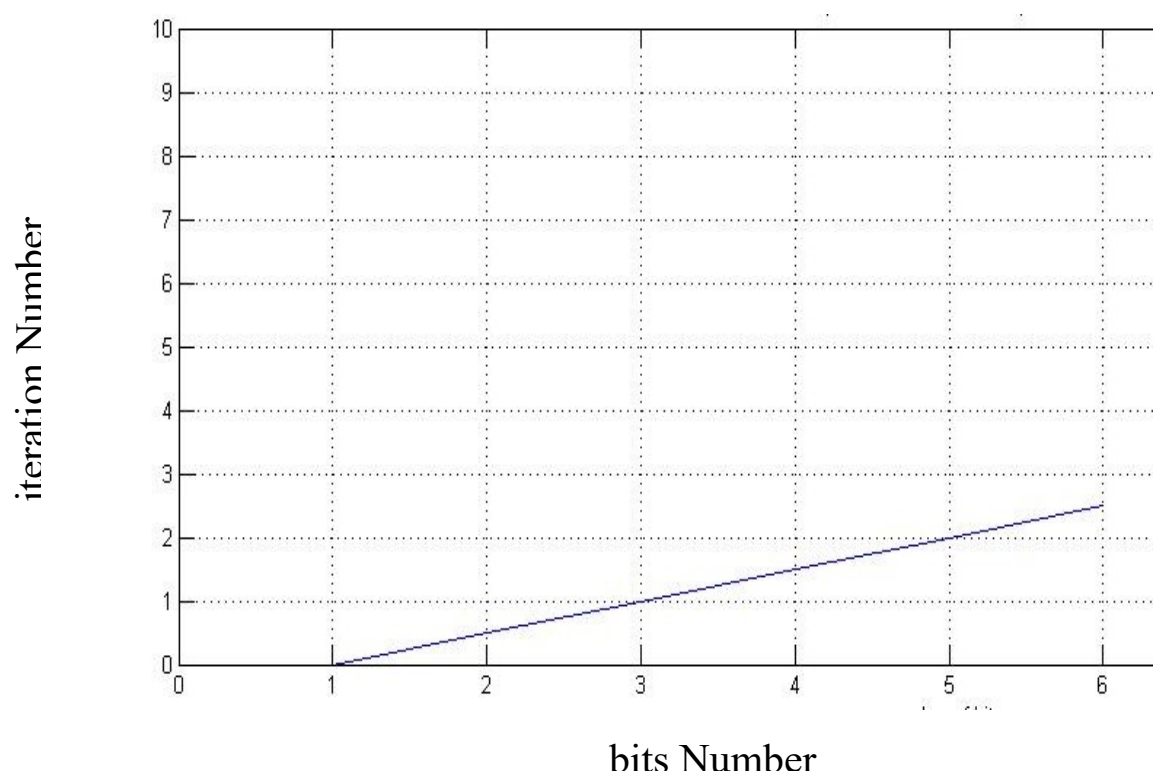

(b)

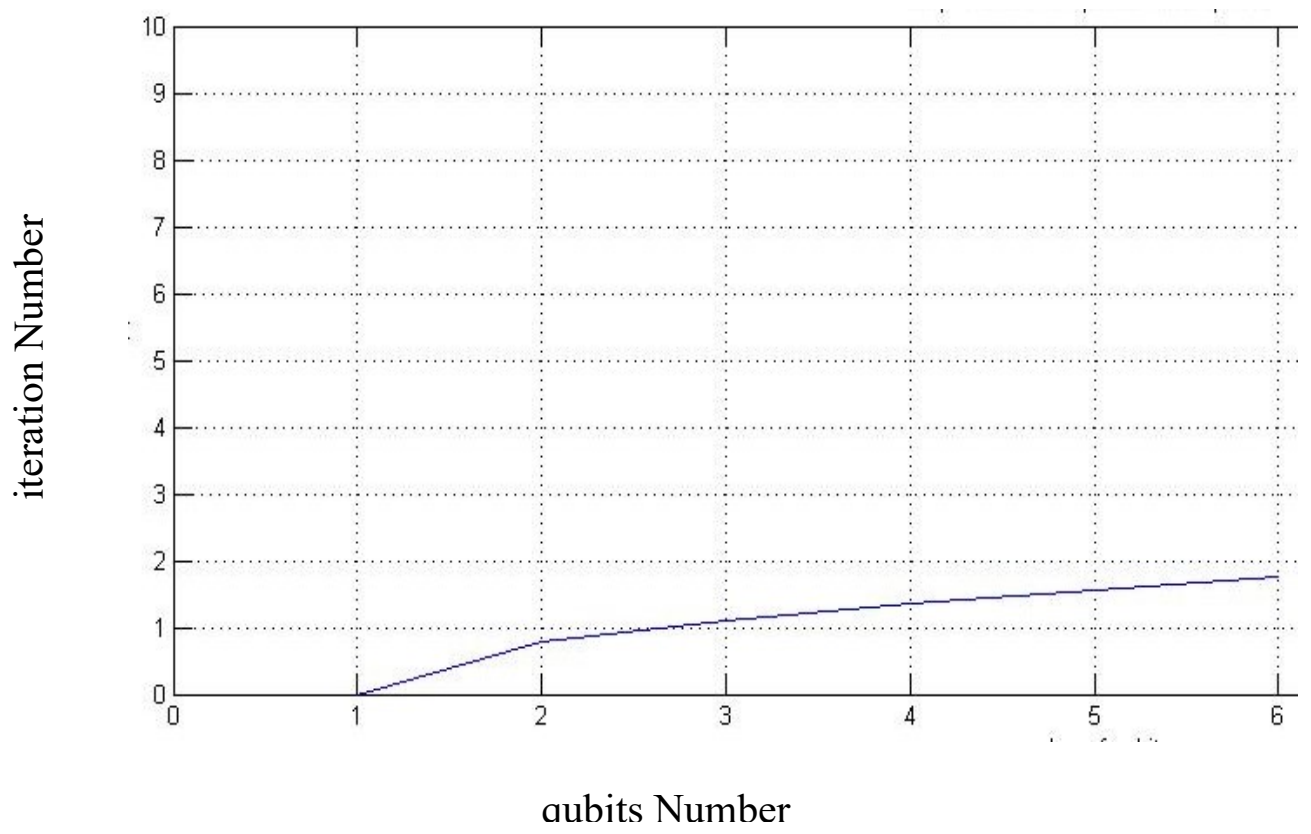

Figure(7) : iteration required (a) classical process (b) quantum process 
For clarification ten (qubits and bits) of information have been taken and tested as shown in figure(8). The processing iteration required in the classic rose by half than it was in five qubits, while the increase in quantum iteration very few and this difference increased exponentially whenever increasingly in unite of information (qubits and bits).

(a)
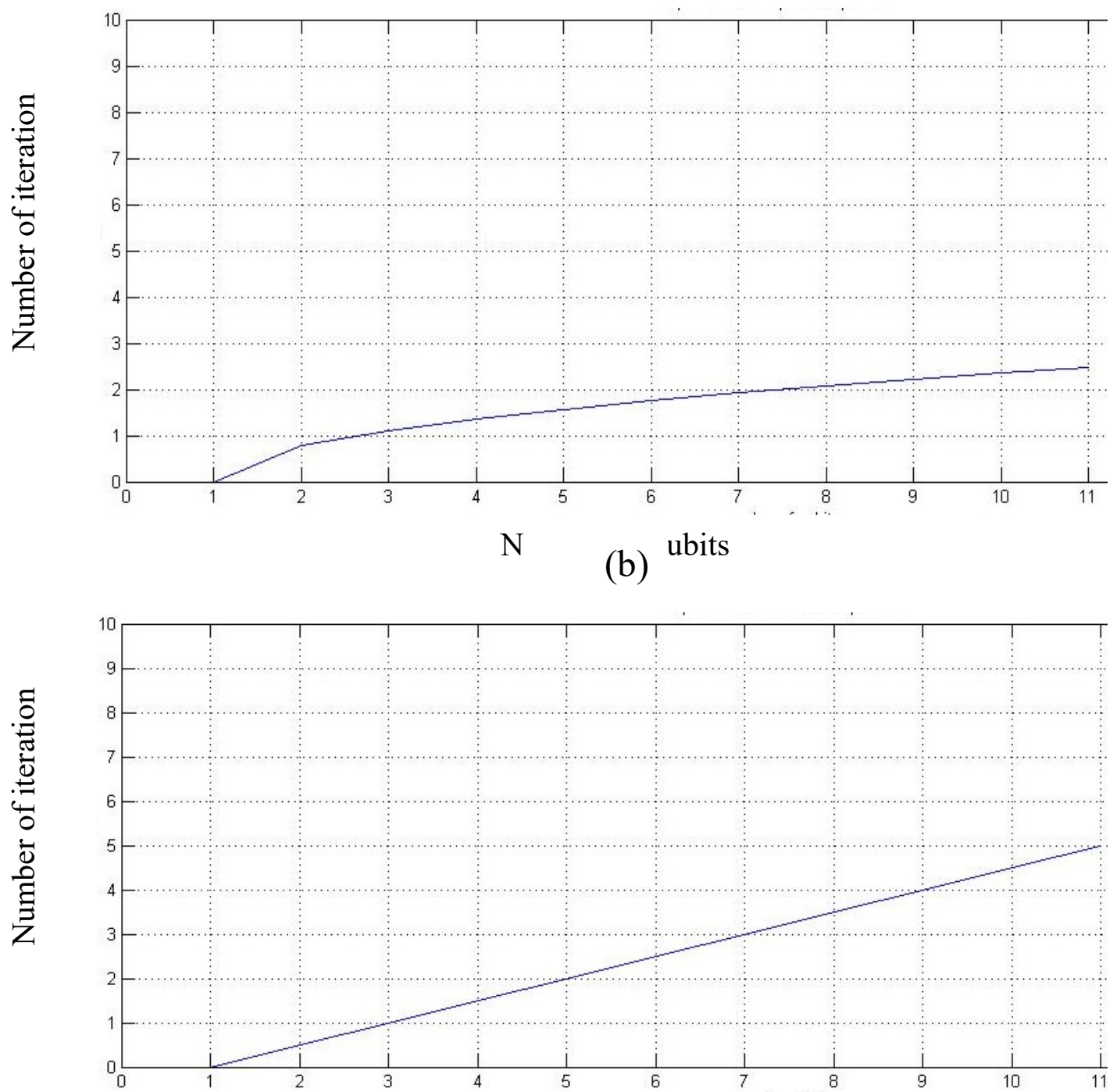

Number of bits

Fig.(8) : iteration required for ten unit of information (bits and qubits)

(a) classical process (b) quantum process 


\section{Conclusion:}

From result, it can be concluded that, the facial recognition have been improved when using the proposed algorithm that the recognition of the patterns becomes faster than the classical processing. Also in this work huge data can be managed and processing without increasing in Hardwar (like memory storage or mlti-processor ect.), as quantum computer realized as a revolution in image processing and huge data management.

\section{Reference:}

[1] Santaji Ghorpade, Jayshree G., Shamla Mantri. (Dec 2010):'Pattern Recognition using Neural Networks", International journal of computer since and information technology, ISSN- 0975-9646, vol 2, No. 6, pp92-98.

[2] M. A. Nielsen \& I. L. Chuang," Quantum Computation and Quantum Information", the press syndicate of the University of Cambridge, United Kingdom, 2000.

[3] L. K. Grover, "A fast quantum mechanical algorithm for database search ",STOC, Page 212-219,USA,1996.

[4] A. Y. Vlaso," Quantum Computations and Images Recognition”, arXiv:quant$\mathrm{ph} / 9703010$ (1997).

[5] R. Schützhold, "Pattern recognition on a quantum computer",Phy. Rev. A 67(6), 062311 (2003).

[6] Beach, G.,Lomont, C., Cohen, C. " Quantum Image Processing (QuIP)", Proc. Appl. Imagery Pattern Recognit, Workshop, 39-44 (2003).

[7] Venegas-Andraca, S.E., Bose, S.," Storing, processing and retrieving an image using quantum mechanics",Proc. SPIE Conf. Quantum Inf. Comput. vol. 5105, 137-147 (2003).

[8] Venegas-Andraca, S.E.," Discrete Quantum Walks and Quantum Image Processing", Thesis submitted for the degree of Doctor of Philosophy at the University of Oxford (2005).

[9] Venegas-Andraca, S.E., Ball, J.L.," Processing images in entangled quantum systems",Quantum Information Processing. 9 (1), 1-11 (2010).

[10] Latorre, J.I.," Image compression and entanglement", arXiv:quant-ph/0510031 (2005).

[11] Le, P.Q., Dong, F., Hirota, K., ” A flexible representation of quantum images for polynomial preparation, image compression, and processing operations", Quantum Inf. Process.10(1),63-84(2011).

[12] Le, P.Q., Iliyasu, A.M., Dong, F., Hirota, K.," Efficient color transformations on quantum images", J.Adv. Comput. Intell. Intell. Inf. 15(6), 698-706(2011). [13]M. Mastriani,"Quantum image processing",DLQSLLC , 4431NW63RD Drive , Coconut Creek, FL 33073,USA. 
[14] Le, P.Q., Iliyasu, A.M., Dong, F.Y., Hirota, K., "Strategies for designing geometric transformations on quantum images", Theoretical Computer Science.412(15),15061418(2011).

[15] Srivastava, M., Panigrah, P.K.," Quantum Image Representation Through TwoDimensional Quantum States and Normalized Amplitude", arXiv: quant$\mathrm{ph} / 1305.2251(2013)$.

[16] Fei Yan , Abdullah M. Iliyasu and Zhengang Jiang ,:( Quantum ComputationBased Image Representation, Processing Operations and Their Applications), Entropy 2014, 16, 5290-5338; doi: 10.3390/e16105290.

[17] AT\&T Laboratories Cambridge, The database of faces at http://www.cl.cam.ac.uk/research/dtg/attarchive/facesataglance.html. 